\title{
Is there Room for Geoengineering in the Optimal Climate Policy Mix?
}

\author{
O. Bahn*1, M. Chesney ${ }^{2}$, J. Gheyssens ${ }^{2}$, R. Knutti ${ }^{3}$, and A.C. Pana ${ }^{2}$ \\ ${ }^{1}$ GERAD and MQG, HEC Montréal, Montréal (Qc), H3T 2A7, Canada \\ ${ }^{2}$ Institute of Banking and Finance, University of Zurich, Zurich, Switzerland \\ ${ }^{3}$ Institute for Atmospheric and Climate Science, ETH Zurich, Zurich, Switzerland
}

May 28, 2014

\begin{abstract}
We investigate geoengineering as a possible substitute for adaptation and mitigation measures to address climate change. With the help of an integrated assessment model, we distinguish between the effects of solar radiation management on atmospheric temperature levels and its side-effects on ecosystems. To address the uncertainty regarding the magnitude of side-effects, we rely on a distributional analysis. Our results indicate that mitigation is the preferred strategy, with adaptation acting as an effective complement. As geoengineering brings significant side-effects on the environment, it is used in only a few of the analyzed scenarios. We then discuss additional concerns with geoengineering, and analyze their impacts on policy choices. In particular, we account for the possibility to make wrong assumptions about side-effects.

Keywords: Climate change; Climate policy mix; Adaptation; Mitigation; Geoengineering

JEL: Q43, Q48, Q54, Q58.
\end{abstract}

\section{Introduction}

Despite international initiatives to curb greenhouse gas (GHG) emissions, atmospheric concentrations keep increasing. In this context, alternatives (or complements) to the traditional mitigation approach are considered. The first such strategy is adaptation. It covers a large array of sectors and options, and can be 'proactive' or 'reactive'. Despite having gained attention for its advantages, investments in adaptation remain quite limited.

Given the increasing risk of an unmanageable temperature path, some scientists are advocating a second alternative to mitigation, namely geoengineering solutions. They

*olivier.bahn@hec.ca 
correspond to deliberate modifications of the climate system in order to alleviate climate change impacts (Keith, 2000). One may distinguish between two main techniques, namely Carbon Dioxide Removal (CDR) and Solar Radiation Management (SRM).

In this paper, we focus on a SRM approach that targets the reduction of incoming solar radiation by injection of sulfur in the stratosphere, believed to be one of the most efficient geoengineering strategies to reduce global temperature (Wigley, 2006; Shepherd et al., 2009). Its premise is the ability to keep temperature levels artificially low, instead of reducing GHG emissions. In case of abrupt climate changes, with rare but catastrophic impacts, SRM could act as a quick and effective temperature 'backstop' (Barrett, 2008), while adaptation and mitigation measures could have only limited effectiveness.

However, SRM brings along important risks, as it may produce unintended consequences and harmful side effects (Victor, 2008). Injecting sulfur particles into the upper atmosphere is expected to cause ozone depletion (Crutzen, 2006; Tilmes et al., 2008), alter ecosystems (Stanhill and Cohen, 2001), and trigger regional imbalances (e.g., in the patterns of surface temperature, radiation, and the water cycle; Bala et al., 2008; Niemeier et al., 2013; Kravitz et al., 2013; Schaller et al., 2013). Furthermore, SRM achieves only an 'artificial' reduction in temperature levels. With a continued increase in concentrations, the injection of aerosols would need to raise proportionally, and a disruption would lead to a significant jump in temperatures at the corresponding concentration level (Brasseur and Roeckner, 2005; Lenton and Vaughan, 2009; Vaughan and Lenton, 2012) with probable dire consequences.

Given these important caveats, support for geoengineering measures has been inconclusive so far. Assuming low side-effects, Crutzen (2006), Wigley (2006), and Carlin (2007) recommend this strategy, but with a strong warning. Mitigation emerges then as the safe and ethical solution, while geoengineering should be backed by additional research. Recent studies focus on modeling decision-making in the context of multiple sources of risk. Gramstad and Tjotta (2010) propose an integrated assessment model (IAM) that allows for parametric uncertainty in the impact of sulfur dissipation on radiative forcing. They find that SRM passes the cost-benefit test under all scenarios, but admit that other factors (such as the risk of SRM interruptions) could lead to the rejection of such projects. Using a different IAM, Goes et al. (2011) account for the failure to sustain aerosol forcing and for the subsequent unraveling of drastic climate changes. In such a case, SRM is found to be an economically inefficient strategy.

In this paper, we contribute to the geoengineering debate with the Ada-BaHaMa integrated assessment model (Bahn et al., 2012), enriched to explicitly consider a SRM strategy. We distinguish between the different effects of SRM on atmospheric temperature levels and ecosystems. While the desired effects of SRM on radiative forcing can be estimated with a significant degree of confidence (Crutzen, 2006), the undesired sideeffects of sulfur dissipation represent a large and important unknown. We focus on this second uncertainty source, and use a distributional analysis based on a binomial tree representation of possible evolutions of side-effects over time. Our original contribution consists in assessing, within an integrated assessment framework, the optimal policy mix when different strategies are available (mitigation, proactive and reactive adapta- 
tion, and SRM) while accounting for the downside risks of SRM.

The reminder of the paper is structured as follows. Section 2 details our dynamic IAM, whose calibration is presented in Section 2.2. Sections 3 and 4 provide numerical results and analyze specific uncertainties related to SRM. Section 5 concludes.

\section{Modeling approach}

\subsection{Overview}

In this section we briefly review the original Ada-BaHaMa model (Bahn et al., 2012) and detail the new modeling features: i) the introduction of SRM as an instrument to control temperature increase and ii) a separate accounting of proactive and reactive adaptation.

The Ada-BaHaMa model distinguishes between two types of economy: a 'carbon' economy (our present economy), where production is fossil fuel intensive, and a 'lowcarbon' economy with small GHG contributions. More precisely, production $(Y)$ occurs in the two economies according to an extended Cobb-Douglas function in three inputs: capital $(K)$, labor $(L)$, and energy (measured through GHG emissions $E$ ). Capital stock in each economy evolves according to the choice of investment $(I)$ and a standard depreciation relation. Total labor $(L)$ is divided between the two economies.

\subsubsection{Climate dynamics in presence of SRM}

GHG stocks evolve according to the dynamic equations of the DICE model (Nordhaus, 2008) that distinguishes between three reservoirs: i) an atmospheric one $\left(M_{A T}\right)$, ii) a quickly mixing one in the upper oceans and the biosphere $\left(M_{U P}\right)$, and iii) a slowly mixing deep-ocean reservoir $\left(M_{L O}\right)$ which acts as a long-term sink.

We extend the original Ada-BaHaMa to account for a geoengineering strategy that increases the albedo effect through sulfur injection in the stratosphere (SRM). This directly affects the relationship between the accumulation of GHGs and the temperature deviation, by reducing the radiative forcing $F(t, s)$ :

$$
F(t, s)=\eta \log _{2}\left(\frac{M_{A T}(t, s)}{M_{A T}(1750)}\right)+F_{E X}(t)-\omega G(t, s)
$$

where $\eta$ is a calibration parameter for a climate sensitivity of $3^{\circ} \mathrm{C}, F_{E X}$ the exogenous radiative forcing term, $\omega$ the effectiveness factor of SRM, and $G$ the amount of sulfur injected in the stratosphere measured in teragrams of sulfur $(\mathrm{Tg} \mathrm{S})$. The index $s$ identifies the specific 'side-effects' scenario that describes the evolution of the damage level from the uncertain SRM strategy.

The other elements of the climatic model remain unchanged from Ada-BaHaMa and follow the DICE model to compute the earth's mean surface temperature $\left(T_{A T}\right)$ and the average temperature of the deep oceans $\left(T_{L O}\right)$. 


\subsubsection{Climate change damages and SRM side-effects}

Ada-BaHaMa follows the approach used in MERGE (Manne and Richels, 2005) to link climate change damages and their economic impacts. Here, we additionally account for negative externalities from sulfur injection. According to Ramanathan et al. (2001) and Brovkin et al. (2009), dissipating large amounts of sulfur in the upper atmosphere may have potentially disruptive effects on weather patterns and the water cycle. Additional damages are expected in case sulfur particles enter the troposphere and add to the sulfur concentration in soil (Crutzen, 2006). Nevertheless, the exact type and magnitude of side-effects remain unknown, and our modeling is not immune to these uncertainties.

We model the magnitude of SRM negative side-effects $\left(D_{G E}\right)$ based on: i) the amount of sulfur used relative to the natural stock of sulfur observed in the stratosphere in 1750 , and ii) a stochastic factor quantifying in economic terms the possible side-effects. The damages persist over time and exhibit the following dynamics:

$$
D_{G E}(t+1, s)=\delta D_{G E}(t, s)+\alpha_{G E}(t+1, s) \frac{G(t+1, s)}{S G_{n a t}}
$$

where $\delta(<1)$ is a constant depreciation rate, $S G_{n a t}$ the natural $(1750)$ concentration level of sulfur in the upper atmosphere, and $\alpha_{G E}$ a time-varying random process. Each state $s$ describes a different and unique path taken by $\alpha_{G E}(t, s)$ between $t=0$ and $t=T$ (the model horizon). In total, more than 32,000 different paths were used to construct the distribution of the SRM damages; see Section 2.2. Note that Eq. (2) accounts only for 'direct' effects in the same time period (such as changes in the water cycle). 'Indirect' effects (such as ocean acidification) are addressed in Section 4.

The temperature increase $\left(T_{A T}\right)$ entails damages that can be alleviated through adaptation $(A D)$. They impact the production function together with SRM side-effects. We model the total economic loss factor (ELF) as follows:

$$
\operatorname{ELF}(t, s)=1-\left(\operatorname{AD}(t, s)\left(\frac{T_{A T}(t, s)-T_{d}}{c a t_{T}-T_{d}}\right)^{2}+D_{G E}(t, s)\right)
$$

where $T_{d}$ is the temperature deviation (from pre-industrial level) at which damages start to occur, while cat $_{T}$ represents the 'catastrophic' temperature level at which the entire production would be wiped out. To have a comparable basis with the current literature on IAM with adaptation, $T_{d}$ and $c a t_{T}$ are calibrated to replicate the damage intensity in DICE; see Section 2.2.

\subsubsection{Proactive and reactive adaptation}

Recent IAM models (Bosello et al., 2010; Agrawala et al., 2011) include both reactive and proactive adaptation in the available policy mix. Acknowledging the increased flexibility of considering both strategies, we distinguish between reactive (flow) and proactive (stock) adaptation. We model the effectiveness of the two adaptation strategies in reducing climate change damages as follows:

$$
\mathrm{AD}(t, s)=1-\alpha_{\mathrm{AD}_{p}}(t, s) \frac{K_{3}(t, s)}{K_{3 \max }(t)}-\alpha_{\mathrm{AD}_{r}}(t, s) \frac{S_{3}(t, s)}{S_{3 \max }(t)}
$$


where $\alpha_{\mathrm{AD}_{p}}$ (respectively, $\alpha_{\mathrm{AD}_{r}}$ ) is the maximum proactive (resp. reactive) adaptation effectiveness, $K_{3}$ (resp. $S_{3}$ ) the amount of proactive adaptation capital (resp. reactive adaptation spending), $K_{3 \max }\left(\text { resp. } S_{3 \max }\right)^{1}$ the maximum amount of adaptation capital (resp. spending) that would ensure the optimal effectiveness of the proactive (resp. reactive) adaptation measures. Like $K_{3 \max }$ in our original model, $S_{3 \max }$ is modelled as an increasing function of the temperature level:

$$
S_{3 \max }(t)=\beta_{\mathrm{AD}_{r}}\left(\frac{T_{A T}(t)}{T_{d}}\right)^{\gamma_{\mathrm{AD} r}}
$$

where $\beta_{\mathrm{AD}_{r}}$ and $\gamma_{\mathrm{AD}_{r}}$ are calibration parameters; see Section 2.2.

The two options are assumed to be complementary, in that the implementation of one enhances the effectiveness of the other. This can be justified as follows. On the one hand, reactive adaptation may require the existence of pre-existing (adaptation) infrastructure. On the other hand, it may be used to adjust long-lasting adaptation infrastructure to the newly observed damages. Adaptation effectiveness is modelled as follows:

$$
\begin{aligned}
& \alpha_{\mathrm{AD}_{p}}(t, s)=\left(\overline{\alpha_{\mathrm{AD}_{p}}}-\underline{\alpha_{\mathrm{AD}_{p}}}\right)\left(\gamma_{1} \frac{K_{3}(t, s)}{K_{3 \max }(t)}+\gamma_{2} \frac{S_{3}(t, s)}{S_{3 \max }(t)}\right)+\underline{\alpha_{\mathrm{AD}_{p}}} \\
& \alpha_{\mathrm{AD}_{r}}(t, s)=\left(\overline{\alpha_{\mathrm{AD}_{r}}}-\underline{\alpha_{\mathrm{AD}_{r}}}\right)\left(\gamma_{1} \frac{S_{3}(t, s)}{S_{3 \max }(t)}+\gamma_{2} \frac{K_{3}(t, s)}{K_{3 \max }(t)}\right)+\underline{\alpha_{\mathrm{AD}_{r}}}
\end{aligned}
$$

where $\alpha_{\mathrm{AD}_{p}}$ and $\overline{\alpha_{\mathrm{AD}_{p}}}$ (resp., $\underline{\alpha_{\mathrm{AD}_{r}}}$ and $\overline{\alpha_{\mathrm{AD}_{r}}}$ ) are the minimum and maximum effectiveness values for proactive (resp., reactive) adaptation, and $\gamma_{1}, \gamma_{2}$ calibration parameters $\left(\gamma_{1}>\gamma_{2}, \gamma_{1}+\gamma_{2}=1\right)$. We model adaptation effectiveness such that in particular: i) the absence of one adaptation strategy does not make the other ineffective, but reduces its potential; and ii) only maximum capital level $\left(K_{3}(t, s)=K_{3 \max }(t)\right)$ and spending $\left(S_{3}(t, s)=S_{3 \max }(t)\right)$ ensure maximum effectiveness.

\subsubsection{Welfare maximization}

As with the original Ada-BaHaMa model, we maximize social welfare $(W)$ given by the sum over $T$ 10-year periods of a discounted utility from per capita consumption. This maximization is done independently for each state $s$, corresponding to a certain evolution of SRM side-effects. Consumption comes from an optimized share of production, the remaining being used: i) to invest in the production capital of the carbon and low-carbon economies $\left(I_{1}, I_{2}\right)$ and in the proactive adaptation capital $\left(I_{3}\right)$; ii) to spend for reactive adaptation $\left(S_{3}\right)$ and SRM measures $\left(S_{4}\right)$; and iii) to pay for energy costs. The presence of damages (defined by the ELF factor) reduces the available production such that:

$$
\begin{aligned}
\operatorname{ELF}(t, s) Y(t, s)= & C(t, s)+I_{1}(t, s)+I_{2}(t, s)+I_{3}(t, s)+S_{3}(t, s)+S_{4}(t, s) \\
& +p_{E_{1}}(t, s) \phi_{1}(t, s) E_{1}(t, s)+p_{E_{2}}(t, s) \phi_{2}(t, s) E_{2}(t, s)
\end{aligned}
$$

\footnotetext{
${ }^{1}$ We impose at all time periods $K_{3}(t, s) \leq K_{3 \max }(t)$ and $S_{3}(t, s) \leq S_{3 \max }(t)$.
} 


\subsection{Calibration}

The economy and climate modules are calibrated on the 2007 version of DICE, hereafter DICE20072. We calibrate a baseline scenario - in which only the carbon economy is producing - to match as closely as possible production, concentration, and temperature trajectories of the DICE2007 baseline. Production in the low-carbon economy is more energy efficient, but also more costly, than in the carbon economy. Without distinguishing among specific technologies, we rely on the MERGE model (Manne and Richels, 2005) for its calibration.

Calibration of the adaptation strategies follows the AD-DICE approach (de Bruin et al., 2009), accounting for the additional reactive adaptation option and its complementarity with proactive adaptation. The calibration of $K_{3 \max }$ and $S_{3 \max }$ relies on World Bank estimates (Margulis and Narain, 2009). However, we assume that reactive adaptation is $50 \%$ more costly than proactive adaptation to achieve its maximum potential ${ }^{3}$. The calibration of the effectiveness parameters $\alpha_{\mathrm{AD}_{p}}$ and $\alpha_{\mathrm{AD}_{r}}$ reflects stylized assumptions about the reactive-proactive relationship. First, we assume that reactive adaptation is slightly more efficient than proactive adaptation, as 'last-minute' strategies are easier to adjust to observed damages. This assumption is coherent with Agrawala et al. (2011), where reactive adaptation offsets on average $27 \%$ of gross damages and proactive adaptation only $21 \%$. Our calibration is very close, with $25 \%$ for reactive adaptation $\left(\overline{\alpha_{\mathrm{AD}_{r}}}\right)$ and $22 \%$ for proactive $\left(\overline{\alpha_{\mathrm{AD}_{p}}}\right)$. Second, the maximum effectiveness of total adaptation cannot be higher than 0.5. This is coherent with Nordhaus and Boyer (2000) where total adaptation potential is 0.48 (compared to 0.47 in our calibration). And third, the difference between minimum and maximum effectiveness for the two strategies is chosen to be relatively small, which implies that proactive and reactive adaptation options are only weakly complementary, similar to Agrawala et al. (2011).

The values for the different SRM parameters are derived from Ramanathan et al. (2001), Crutzen (2006), and Shepherd et al. (2009). For the sulfur effectiveness $(\omega)$ we rely on Crutzen (2006) that reports (from the Mount Pinatubo natural experiment) a "sulfate climate cooling efficiency of $0.75 \mathrm{~W} / \mathrm{m}^{2}$ per $\mathrm{Tg} S$ in the stratosphere". The lack of scientific evidence regarding possible SRM externalities motivates us to rely on scenario analysis for side-effects, where many possible paths are considered. To calibrate $\alpha_{G E}$, we define three levels of side-effects: an initial level $\alpha_{G E}\left(t_{0}\right)$, and two boundary values for the final period $T$ defining a minimum $\left(\underline{\alpha_{G E}}\right)$ and a maximum level $\left(\overline{\alpha_{G E}}\right)$. To relate SRM side-effects to climate change damages, we tentatively set $\alpha_{G E}\left(t_{0}\right)$ to 0.2523 , such that the injection of $1 \mathrm{Tg} \mathrm{S}$ per year during one decade matches the damages from a temperature increase of one-fourth of the catastrophic temperature deviation $\left(\mathrm{cat}_{T}\right)$. Similarly, $\overline{\alpha_{G E}}$ is set to 1.0366 , resulting in side-effects equivalent to damages for $0.5 \mathrm{cat}_{T}$. The uncertainty regarding the evolution of $\alpha_{G E}$ over time is modeled using a binomial tree approach, with the following dynamics:

\footnotetext{
${ }^{2}$ See: http://www.econ.yale.edu/ nordhaus/DICE2007.htm.

${ }^{3}$ Indeed, deployment of last minute strategies should incur organizational costs much higher than under a long-planned strategy. Besides, compared to proactive adaptation, reactive adaptation should bear some of the infrastructure and deployment costs upfront, which induces large overhead.
} 


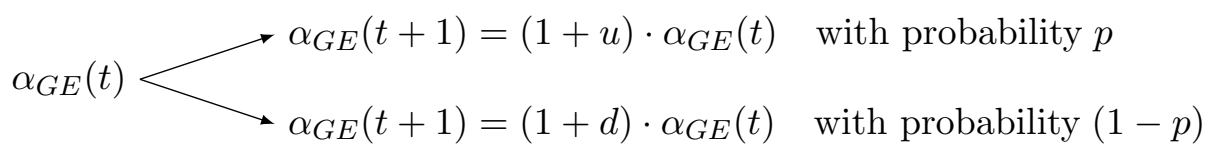

with $u$ the percentage increase, $d$ the percentage decrease, and $p$ the probability of having an up-move next period. We design a symmetric tree: $p=0.5$ and $d=-u$. Parameter $u$ is calibrated to link $\alpha_{G E}\left(t_{0}\right)$ to $\overline{\alpha_{G E}}$ in a monotonically increasing path over 15 decades (until $T=2155$ ): $\overline{\alpha_{G E}}=\alpha_{G E}\left(t_{0}\right) \cdot(1+u)^{15}$. Based on the binomial representation we obtain $32,768\left(=2^{15}\right)$ different but equiprobable scenarios. This time-varying representation of SRM side-effects is motivated by the view that ecosystems present dynamic and non-linear resilience to shocks and perturbations (Holling, 1973; Gunderson, 2003). Complex socio-ecological systems can have a highly optimized tolerance to a certain set of disturbances (Carlson and Doyle, 2002; Janssen et al., 2007), but they would suffer if the disturbances evolve or change outside of their optimized tolerance zone, causing them to move to new equilibria. Considering the multitude of systems that SRM impacts and the likely existence of complex feedback loops, we consider its short- and long-term disturbances to be unpredictable and to evolve in a possibly non-monotonic manner. We therefore proxy the evolution of ecosystem damages with a binomial tree such that at each period, the process can evolve to a weaker or stronger state.

\section{Optimal policy mix}

\subsection{Selected scenarios for SRM side-effects}

In this section, we consider only three scenarios for SRM side-effects. The first scenario refers to strong damages, where $\alpha_{G E}$ increases monotonically to $\overline{\alpha_{G E}}$. It corresponds to the upper edge of the distribution (of all possible paths for side-effects) when all forecasted impacts would be triggered (ozone depletion, unfavorable change in precipitation patterns, warming of the tropical tropopause) and the total magnitude would be amplified by cross-interactions. The second scenario corresponds to a mild scenario, where $\alpha_{G E}$ evolves non-monotonically to reach at $T$ a value ${ }^{4}$ between $\alpha_{G E}$ and $\overline{\alpha_{G E}}$, when only some of the expected damages occur. The third scenario refers to weak damages, where $\alpha_{G E}$ decreases monotonically to $\alpha_{G E}$. It corresponds to the lower edge of the distribution when sulfur dissipation has almost no side-effects.

In these three scenarios, all policy strategies (mitigation, adaptation, and SRM) are available. We consider two additional policy scenarios: a mitigation-only scenario (where adaptation and SRM are not available) and an adaptation-mitigation scenario (where SRM is not available).

The traditional policy to address climate change is mitigation, which corresponds in our model to a transition from the carbon economy to the low-carbon one. In the

\footnotetext{
${ }^{4}$ Equal to 0.1125 , about two times the value of $\underline{\alpha_{G E}}$. It has been chosen to get an illustrative scenario where all the policy options are used.
} 
mitigation-only scenario, there is a clear transition between the two economies: the carbon-intensive capital is rapidly phased out after 2055 and completely replaced by the low-carbon capital by the end of the century. When adaptation is also available as a policy tool (adaptation-mitigation scenario), this transition does take place, but starts one decade later. The SRM option has contrasted implications: under mild side-effects, the transition is further delayed by ten years (after 2075), whereas the transition never occurs under weak side-effects. In agreement with Barrett (2008), our results indicate thus that there is no incentive to curb GHG emissions if SRM is available and its sideeffects are benign.

Fig. 1 captures adaptation and SRM decisions. When its side-effects are weak, SRM is the main instrument to address climate change. It is used after 2055 (substituting for mitigation efforts), together with adaptation (after 2065). When its side-effects are mild, SRM is only used (after 2075) as a complement to adaptation and mitigation strategies. SRM is not used when its side-effects are strong. Concerning adaptation, we note that: i) the accumulation of proactive adaptation capital starts before spending on reactive adaptation; and ii) the decreasing trend in adaptation towards the end of the horizon reflects the lowering of temperature (see Fig. 2) reached through SRM and/or mitigation.
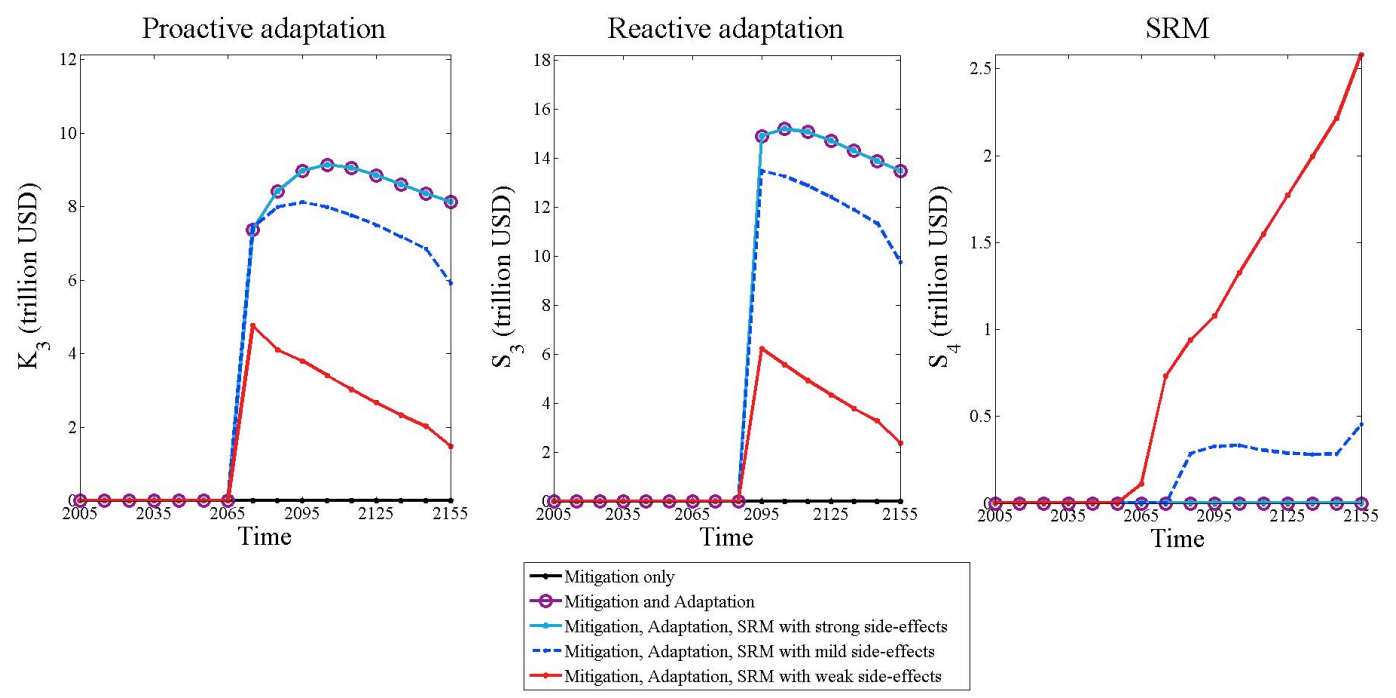

Figure 1: Capital accumulation in proactive adaptation, and decade spending with reactive adaptation and SRM

An interesting aspect is the relative dynamics of reactive adaptation and SRM. As ex-post measures, they share the advantage of rapid implementation and immediate reduction of damages. The weight given to either measure depends on the magnitude of sulfur damages: i) in case of strong side-effects, between the two, only adaptation is used; ii) for mild and weak side-effects, both measures enter the policy portfolio, with 
more weight given to SRM as its side-effects decrease. This results from the limited effectiveness of reactive adaptation.

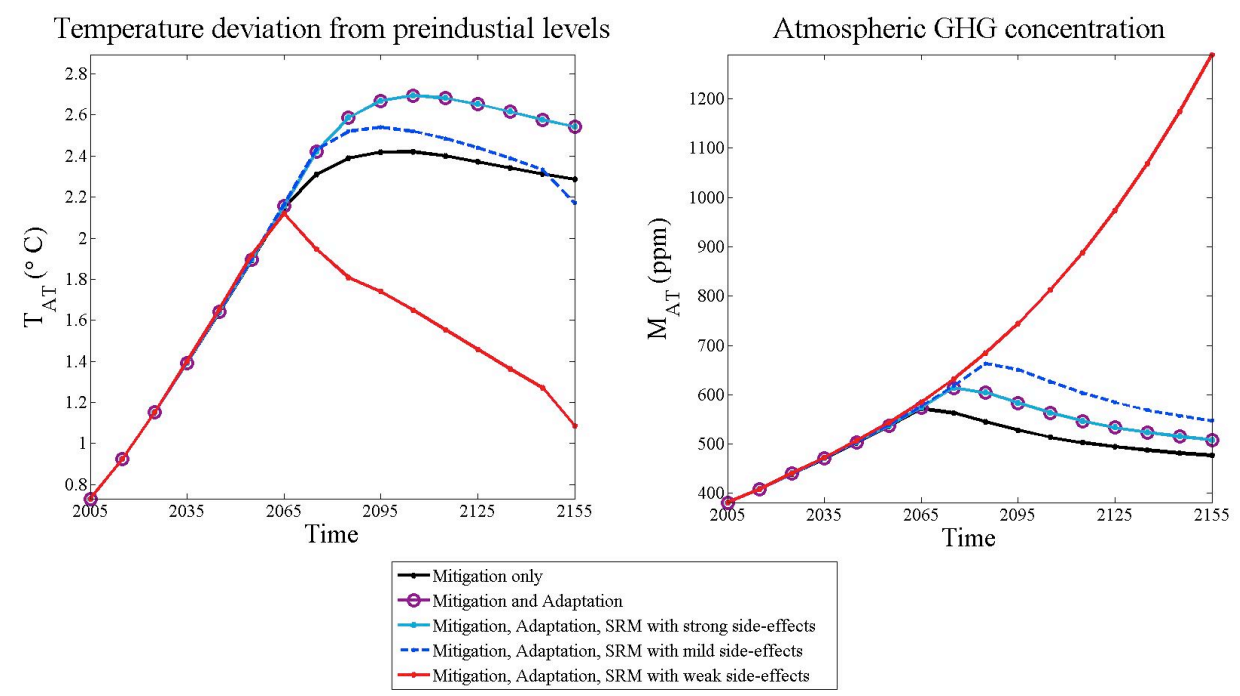

Figure 2: Atmospheric temperature and GHG concentrations

Fig. 2 reveals that temperature is kept below the $2{ }^{\circ} \mathrm{C}$ threshold proposed by the Copenhagen Accord (except for a brief period) only when SRM side-effects are weak. This is achieved at the expense of: i) a large deployment of SRM; and ii) high GHG concentrations, due to the absence of any mitigation. In the other scenarios, concentrations and then temperature decrease with the transition to the low-carbon economy.

Imposing the $2{ }^{\circ} \mathrm{C}$ limit yields an earlier implementation of mitigation and/or SRM. With strong side-effects, investments in mitigation are advanced by two decades (2035); with low side-effects, SRM remains predominant and is implemented from 2065 on. To reduce the lower climate change damages, adaptation is still used with a similar timing (compared to the unconstrained temperature cases) but requires less funding.

\subsection{Distributional analysis for SRM side-effects}

In this section, we examine policy decisions for all the side-effect scenarios from the binomial tree representation; see Section 2.2. Fig. 3 illustrates two historical distributions of the average ${ }^{5}$ value of side-effects along each path. The first histogram (in gray) corresponds to the scenarios where SRM is used, the second (in blue) where it is not.

SRM is not used when side-effects are above a given threshold, here when the average $\alpha_{G E}$ is above 0.25 . This value coincides with the initial side-effect level $\left(\alpha_{G E}\left(t_{0}\right)\right)$ and indicates that SRM can only be used at future periods if side-effects become smaller. For the range $[0.18,0.25]$ the two histograms overlap, suggesting that the use of SRM

${ }^{5}$ The average is computed as $\sum_{t=2005}^{2155} \alpha_{G E}(t, s)$, for each scenario $s \in[1,32768]$. 


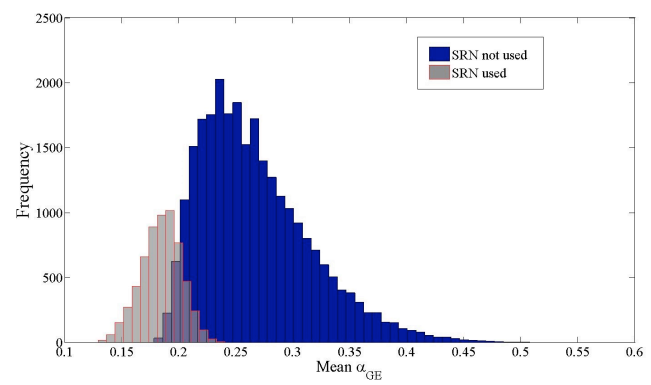

Figure 3: Historical distributions of SRM side-effects

depends not only on the average side-effect value, but also on the minimum value reached at a given period. SRM is only used in $18.7 \%$ of the simulated cases (for at least one decade), at the earliest in 2065. It starts on average around 2090 and lasts for about 45 years.

In $95 \%$ of the cases mitigation is the main policy option. In the remaining $5 \%$, it is fully substituted by SRM. Besides, SRM acts as a complement to mitigation in $14 \%$ of the cases, when mean $\alpha_{G E}$ values are in the $[0.16,0.24]$ range. Adaptation is used in all scenarios, with reactive adaptation implemented on average two decades after proactive. When mitigation is used, adaptation serves mainly to reduce climate change damages, because of the lag between mitigation implementation and temperature reduction. Whereas in the presence of SRM, it serves mainly to compensate SRM sideeffects.

\section{Indirect and unexpected SRM side-effects}

The previous section indicates that SRM should be used when its side-effects are low. Here, we discuss additional concerns with SRM and analyse the adjustments in the optimal policy mix.

An important concern with SRM is the potential damage that would be caused by a failure to sustain the sulfur dissipation (Robock et al., 2009; Goes et al., 2011). We analyze, in the case of weak side-effects, the consequences of SRM interruption (e.g., following a technical breakdown) from 2105. Our results confirm that temperature increases rapidly after the SRM failure, and that the benefits to be gained from mitigation are then limited due to inertia in the climate system.

Next, our model accounts for direct SRM side effects; see again Eq. (3). But scientists point also to indirect side-effects such as ocean acidification (Orr et al., 2005). This arises from high $\mathrm{CO}_{2}$ concentrations in the absence of mitigation. Damages to ocean ecosystems may occur with $\mathrm{CO}_{2}$ levels as low as $450 \mathrm{ppm}$ (Cao and Caldeira, 2008). In our distributional analysis, no scenario is consistent with such a level. In particular, when SRM is used, around $50.3 \%$ of the cases reach a level between 550 and $650 \mathrm{ppm}$, 
the remaining cases are above $650 \mathrm{ppm}$. When constraining for instance concentration levels to $550 \mathrm{ppm}$, the use of SRM is reduced to only $9.5 \%$ of all scenarios (compared with $18.7 \%$ ).

Finally, the most important concern with SRM is probably that the type and magnitude of side-effects remain largely unknown. Up to now, the optimal policy mix was evaluated via scenario analysis, assuming that side-effects were known along each scenario. Here, we study the consequences of incurring a different (random) side-effect path than the one presumed. First, for a given side-effect scenario $s$, we compute following Eq. (2) the presumed SRM damages $\left(D_{G E}(t, s)\right)$ that translate into GDP losses. Next, we compute unexpected GDP losses $\left(D_{G E}\left(t, s^{\prime}\right)\right)$ that would arise if a different side-effect path $s^{\prime}$ occurs. More precisely, we replace in Eq. (2) $\alpha_{G E}(t, s)$ with a factor $\alpha_{G E}\left(t, s^{\prime}\right)$ chosen randomly from the possible side-effect paths, while keeping the same levels for sulfur injection. Forming wrong assumptions regarding the side-effects should only last for short periods. Indeed, although the earth system presents some inertia, the magnitude of side-effects is ex-post identifiable via decreases in production. However, it might take several decades until true realizations are identified. As an illustration, we consider an error horizon of one to three decades and compare GDP losses ${ }^{6}$ under known (presumed) and random (unexpected) side-effect paths. Fig. 4 reveals that GDP losses rise when the side-effect path has a different outcome than the one assumed, even on a ten-year horizon, on average up to two times more across the different scenarios. With a twenty to thirty-year horizon, GDP losses increase further on average, together with their variance and right skewness, indicating that extreme losses have a high chance of occurrence.

Besides estimating welfare losses, we study the adjustments to the optimal policy mix after observing the true damages. As an example, we examine the (extreme) case where side-effects were assumed to be weak, but turn out to be strong. With (presumed) weak side-effects, SRM is first implemented in 2065 at the expense of any mitigation efforts; see again Section 3. But suppose that after three decades the policy maker realizes that side-effects are actually strong. We simulate this situation by running the model from 2065 on with the true $\alpha_{G E}$ values, keeping all investment and spending decisions blocked for the error horizon (2065-2085). The policy maker reacts by stopping all SRM activities from 2095 on. A quick transition to the low-carbon economy takes place, but cannot prevent a temperature increase to almost $3{ }^{\circ} \mathrm{C}$. To alleviate some of the climate damages, adaptation (especially reactive) is also extensively used. Overall, deviating during the error horizon from the optimal policy in case of strong side-effects results in lower consumption levels for the remaining horizon.

\footnotetext{
${ }^{6}$ For each scenario $s \in[1,32768]$, we compute presumed damages as: $\sum_{t=t_{G E}}^{t_{G E}+10 \tau} D_{G E}(t, s)$, where $t_{G E}$ is the first period of SRM implementation in scenario $s$ and $\tau \in\{1,2,3\}$ decades of SRM implementation. Unexpected damages are computed as: $\frac{1}{5000} \sum_{i=1}^{5000} \sum_{t=t_{G E}}^{t_{G E}+10 \tau} D_{G E}\left(t, s_{i}^{\prime}\right)$, where $\alpha_{S E}\left(t, s_{i}^{\prime}\right)$ is randomly selected from the initial side-effect distribution over 5000 simulations.
} 

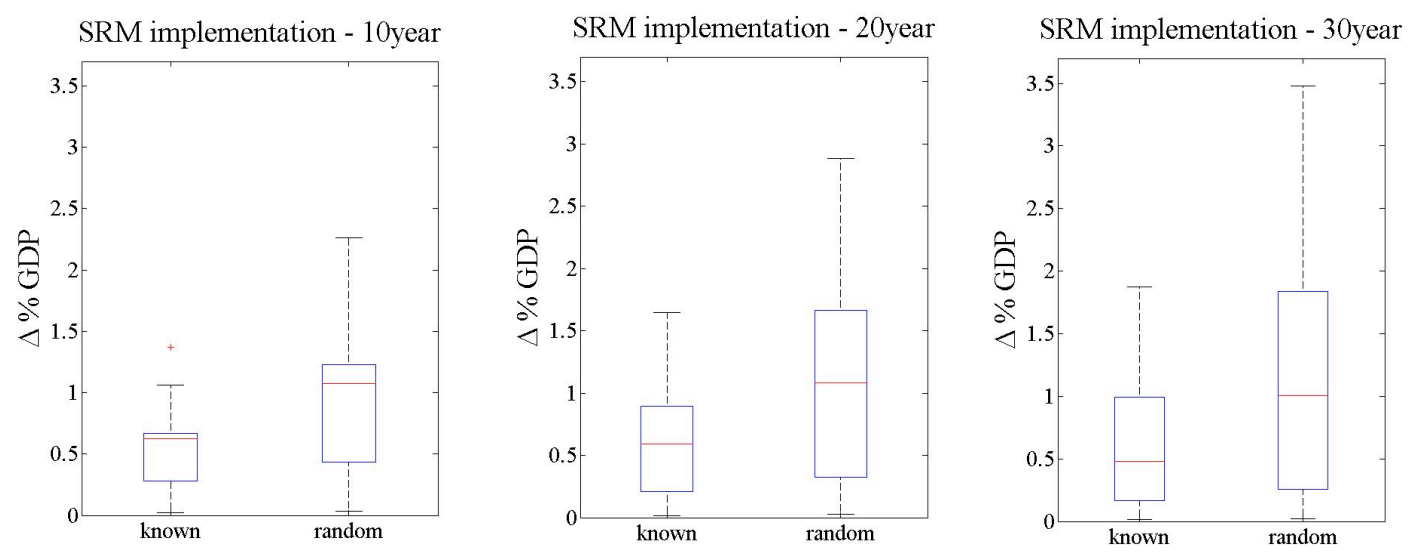

Figure 4: Distribution of GDP losses under known and random SRM damages for different error horizons

\section{Conclusions}

This paper extends the Ada-BaHaMa model with an explicit representation of reactive adaptation and geoengineering (SRM). Reactive adaptation complements proactive adaptation, and its modeling gives a richer representation of possible adaptation strategies. SRM is a potential alternative to traditional climate strategies, and we distinguish between its effects on temperature levels and its side-effects on ecosystems. To address the uncertainty regarding the magnitude of side-effects, we use a distributional analysis based on a binomial tree representation of the evolution of side-effects over time.

We use this new integrated assessment model to analyze the interplay of mitigation, adaptation, and SRM to address global climate change. Our analysis reveals that mitigation is the preferred strategy, with adaptation acting as an effective complement. As SRM entails serious side-effects on the environment, it is used in only $18.7 \%$ of the analyzed scenarios, with different implementation horizons depending on the severity of the side-effects. The implementation of SRM measures is done at the expense of mitigation efforts. At the extreme, under weak side-effects, the transition to a low-carbon economy does not take place (within the model horizon).

An extensive use of SRM brings additional concerns. One is the potential damage that would be caused by a failure to continue with sulfur dissipation. Another one consists in indirect side-effects such as ocean acidification. Since the uncertainty regarding SRM side-effects is currently large, we investigate also possible welfare losses due to incorrect assumptions about these side-effects. Our analysis shows that GDP losses rise when the side-effect path has a different outcome than the one presumed. Besides, 
longer error horizons lead to higher GDP losses, together with increased variance and right skewness, indicating that extreme losses have a higher chance of occurrence.

Our study reinforces the precautionary approach one should have with SRM. Relying in particular on imperfect forecasts for SRM impacts can lead to large welfare losses, even for short implementation horizons. In accordance with Moreno-Cruz and Keith (2013), our findings call thus for the necessity to reduce the uncertainty revolving around SRM before envisioning a full-scale deployment of this strategy.

In our cost-benefit approach, despite the predominant role of mitigation, we note that investments in the low-carbon economy start after 2055 at the earliest. However, when following a cost-effectiveness approach to constrain temperature increase to $2{ }^{\circ} \mathrm{C}$, climate policies are implemented 20 years earlier. Meeting the agreed $2{ }^{\circ} \mathrm{C}$ temperature target would thus require earlier actions at the global level.

Our analysis could be improved along the following lines. First, our model assumes exogenous technological progress. With an endogenous formulation, one might expect that $(\mathrm{R} \& \mathrm{D})$ investments in low-carbon technologies start earlier to get 'on-time' the needed technologies for mitigation. Second, being derived in a cost-benefit framework, our results (and in particular the timing of mitigation) critically depend on the magnitude of the estimated climate change damages, calibrated in our model on DICE and AD-DICE. Recent papers (Stern, 2013; Pindyck, 2013) signal possible underestimations of climate change damages in the DICE-like integrated assessment models; further research could help address these issues. Third, the deployment of SRM depends on various unknowns, such as the type and magnitude of side-effects, and the process governing their variability through time. The calibration of all involved parameters in our model will benefit from revisions reflecting scientific advances. Finally, our model assumes the existence of a well-functioning international cooperation for the deployment of SRM at a global level. Future research could improve our understanding of the SRM option by accounting for differentiated geographical impacts and investigating strategic country behavior. 


\section{References}

Agrawala, S., Bosello, F., et al, 2011. Plan or react? Analysis of adaptation costs and benefits using integrated assessment models. Climate Change Economics 2, 175-208.

Bahn, O., Chesney, M., Gheyssens, J., 2012. The effect of proactive adaptation on green investment. Environmental Science \& Policy 18, 9-24.

Bala, G., Duffy, P.B., Taylor, K.E., 2008. Impact of geoengineering schemes on the global hydrological cycle. Proceedings of the National Academy of Sciences 105 (22), 7664-7669.

Barrett, S., 2008. The incredible economics of geoengineering. Environmental and Resource Economics 39, 45-54.

Bosello, F., Carraro, C., De Cian, E., 2010. Climate policy and the optimal balance between mitigation, adaptation and unavoided damage. Climate Change Economics 1, 71-92.

Brasseur, G.P., Roeckner, E., 2005. Impact of improved air quality on the future evolution of climate. Geophysical Research Letters 32, L23704, doi:10.1029/2005GL023902.

Brovkin, V., Petoukhov, V., et al, 2009. Geoengineering climate by stratospheric sulfur injections: Earth system vulnerability to technological failure. Climatic Change 92, $243-259$.

de Bruin, K., Dellink, R., Tol, R., 2009. AD-DICE: An implementation of adaptation in the DICE model. Climatic Change 95, 63-81.

Cao, L., Caldeira, K., 2008. Atmospheric $\mathrm{CO}_{2}$ stabilization and ocean acidification. Geophysical Research Letters 35, L19609, doi:10.1029/2008GL035072.

Carlin, A., 2007. Global climate change control: Is there a better strategy than reducing green house gas emissions? Univ PA Law Review 155, 128-156.

Carlson, J.M., Doyle, J., 2002. Complexity and robustness. Proceedings of the National Academy of Sciences of the United States of America 99, 2538-2545.

Crutzen, P., 2006. Albedo enhancement by stratospheric sulfur injections: A contribution to resolve a policy dilemma? Climatic Change 77, 211-219.

Goes, M., Tuana, N., Keller, K., 2011. The economics (or lack thereof) of aerosol geoengineering. Climatic Change 109, 719-744.

Gramstad, K., Tjotta, S., 2010. Climate engineering: Cost benefit and beyond. Working papers in economics, University of Bergen, Norway. 
Gunderson, L.H., 2003. Adaptive dancing: Interactions between social resilience and ecological crises. Navigating socialecological systems: Building resilience for complexity and change. Cambridge University Press, Cambridge, UK, 33-52.

Holling, C.S., 1973. Resilience and stability of ecological systems. Annual review of ecology and systematics 4, 1-23.

Janssen, M.A., Anderies, J.M., Ostrom, E., 2007. Robustness of social-ecological systems to spatial and temporal variability. Society and Natural Resources 20, 307-322.

Keith, D., 2000. Geoengineering the climate: History and prospect. Annual Review of Energy and the Environment 25, 245-284.

Kravitz, B., Rasch, P., et al, 2013. An energetic perspective on hydrological cycle changes in the Geoengineering Model Intercomparison Project. Journal of Geophysical Research 118 (23), 13087-13102.

Lenton, T., Vaughan, N., 2009. The radiative forcing potential of different climate geoengineering options. Atmospheric Chemistry and Physics 9, 5539-5561.

Manne, A., Richels, R., 2005. MERGE: An integrated assessment model for global climate change, in: Loulou, R., Waaub, J.P., Zaccour, G. (Eds.), Energy and Environment. Springer. volume 3 of GERAD 25th Anniversary Series, pp. 175-189.

Margulis, S., Narain, U., 2009. The Costs to Developing Countries of Adapting to Climate Change: New Methods and Estimates. Global Report of the Economics of Adaptation to Climate Change Study. The World Bank. Washington, DC.

Moreno-Cruz, J.B., Keith, D.W., 2013. Climate policy under under uncertainty: A case for solar geoengineering. Climatic Change 121, 431-444.

Niemeier, U., Schmidt, H., Alterskjr, K., Kristjnsson, J., 2013. Solar irradiance reduction via climate engineering: Impact of different techniques on the energy balance and the hydrological cycle. Journal of Geophysical Research 118 (21), 11905-11917.

Nordhaus, W., 2008. A Question of Balance. Yale University Press.

Nordhaus, W.D., Boyer, J., 2000. Warning the World: Economic Models of Global Warming. MIT Press (MA).

Orr, J.C., Fabry, V.J., et al., 2005. Anthropogenic ocean acidification over the twentyfirst century and its impact on calcifying organisms. Nature 437, 681-686.

Pindyck, R.S., 2013. Climate change policy: What do the models tell us? Working paper 19244 NBER.

Ramanathan, V., Crutzen, P., Kiehl, J., Rosenfeld, D., 2001. Aerosols, climate, and the hydrological cycle. Science 294, 2119-2124. 
Robock, A., Marquardt, A., Kravitz, B., Stenchikov, G., 2009. Benefits, risks, and costs of stratospheric geoengineering. Geophysical Research Letters 36, L19703, doi:10.1029/2009GL039209.

Schaller, N., Cermak, J., Wild, M., Knutti, R., 2013. The sensitivity of the modeled energy budget and hydrological cycle to $\mathrm{CO}_{2}$ and solar forcing. Earth System Dynamics 4, doi:10.5194/esd-4-253-2013.

Shepherd, J., Caldeira, K., et al., 2009. Geoengineering the climate. Science, governance and uncertainty. Technical Report. The Royal Society.

Stanhill, G., Cohen, S., 2001. Global dimming: A review of the evidence for a widespread and significant reduction in global radiation with discussion of its probable causes and possible agricultural consequences. Agricultural and Forest Meteorology 107, 255-278.

Stern, N., 2013. The structure of economic modeling of the potential impacts of climate change: Grafting gross underestimation of risk onto already narrow science models. Journal of Economic Literature 51(3), 838-859.

Tilmes, S., Mueller, R., Salawitch, R., 2008. The sensitivity of polar ozone depletion to proposed geoengineering schemes. Science 320, 1201-1204.

Vaughan, N., Lenton, T., 2012. Interactions between reducing $\mathrm{CO}_{2}$ emissions, $\mathrm{CO}_{2}$ removal and solar radiation management. Philosophical transactions of The Royal Society $370,4343-4364$.

Victor, D.G., 2008. On the regulation of geoengineering. Oxford Review of Economic Policy 24, 322-336.

Wigley, T., 2006. A combined mitigation/geoengineering approach to climate stabilization. Science $314,452-454$. 